\title{
Properties of Elementary Particle Fluxes in Primary Cosmic Rays Measured with the Alpha Magnetic Spectrometer on the International Space Station
}

\author{
Francesco Nozzoli on behalf of the AMS Collaboration ${ }^{1, a}$ \\ ${ }^{1}$ INFN TIFPA, I-38123 Trento, Italy
}

\begin{abstract}
Precision measurements by AMS of the fluxes of cosmic ray positrons, electrons, antiprotons, protons as well as their rations reveal several unexpected and intriguing features. The presented measurements extend the energy range of the previous observations with much increased precision. The new results show that the behavior of positron flux at around $300 \mathrm{GeV}$ is consistent with a new source that produce equal amount of high energy electrons and positrons. In addition, in the absolute rigidity range $60-500 \mathrm{GV}$, the antiproton, proton, and positron fluxes are found to have nearly identical rigidity dependence and the electron flux exhibits different rigidity dependence.
\end{abstract}

\section{Introduction}

AMS-02 [1-14] is a state-of-the-art particle physics detector designed to operate as an external module on the International Space Station (ISS). It is studying the universe and its origin by searching for antimatter and dark matter, while performing precision measurements of Cosmic Ray (CR) composition and flux.

The high statistics of the measurements, along with the high precision of the experiment, allow to study the detailed variations with rigidity of the flux spectral indices and flux ratios, important in understanding the origin, acceleration and propagation of cosmic rays in our galaxy. In particular, experimental data on the cosmic ray antiprotons, $\bar{p}$, and positrons, $e^{+}$, are crucial for understanding the origin of antimatter in cosmos, providing an insight into new physics phenomena. While some of cosmic ray $\bar{p}$ and $e^{+}$are produced by interactions of the cosmic ray nuclei with the interstellar gas, there could be a substantial contribution from annihilation of dark matter particles, acceleration/production by astrophysical objects, or evaporation of primordial black holes. The sensitivity of cosmic ray positrons to these new phenomena is complementary to the sensitivity of the measurements of antiprotons, whose production by nearby pulsars is expected to be negligible. Therefore AMS has now precisely measured the fluxes of all the elementary particles in the cosmos, this allows for new observations of their properties.

\section{The AMS Detector}

The layout of the AMS detector is shown in Fig.1. It consists of 9 planes of precision silicon Tracker; a Transition Radiation Detector, TRD; four planes of Time of Flight

\footnotetext{
ae-mail: Francesco.Nozzoli@tifpa.infn.it
}

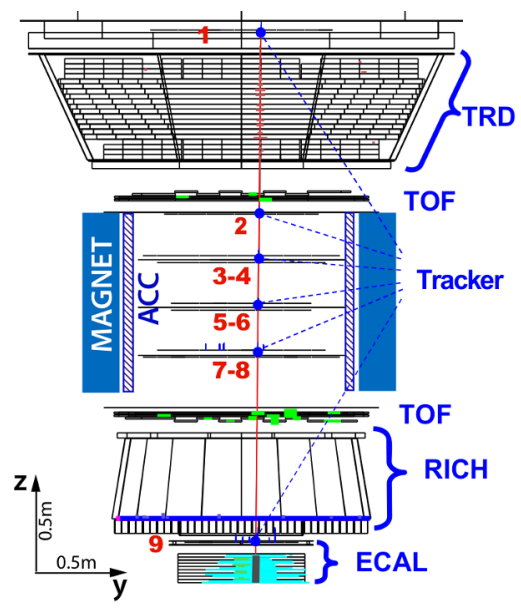

Figure 1. A $868 \mathrm{GeV}$ positron event as measured by the AMS detector on the ISS in the (y-z) plane. Tracker planes 1-9 measure the particle charge, sign and momentum. The TRD identifies the particle as an electron/positron. The TOF measures the charge and ensures that the particle is downward-going. The RICH measures the charge and velocity. The ECAL independently identifies the particle as an electron/positron and measures its energy.

counters, TOF; a Magnet; an array of anti-coincidence counters, ACC, surrounding the inner Tracker; a Ring Imaging Čerenkov detector, RICH; and an Electromagnetic Calorimeter, ECAL. The figure also shows a high energy positron of $868 \mathrm{GeV}$ recorded by AMS. Together, the tracker and the magnet measure charged cosmic rays with momentum $\mathrm{p}$, charge $\mathrm{Z}$ and rigidity $\mathrm{R}=\mathrm{pc} / \mathrm{Ze}$. The tracker has nine layers, the first at the top of the detector, 
the second just above the magnet, six within the bore of the magnet, and the last just above the ECAL. Each layer contains double-sided silicon microstrip detectors that independently measure the $\mathrm{x}$ and $\mathrm{y}$ coordinates. The tracker accurately determines the particle trajectory by multiple measurements of the coordinates with a resolution in each layer of $10 \mu \mathrm{m}$ in the bending (y) direction. The maximum detectable rigidity, MDR, is $2 \mathrm{TV}$ for $|\mathrm{Z}|=1$, $3.2 \mathrm{TV}$ for helium and 3.7 TV for carbon nuclei over the $3 \mathrm{~m}$ lever arm. Signal amplitude from each layer of the tracker also provides an independent measurement of charge $|\mathrm{Z}|$. The overall charge resolution of the tracker, $\Delta \mathrm{Z} / \mathrm{Z}$, is $5 \%$ for $|\mathrm{Z}|$ $=1,3.5 \%$ for helium and $2 \%$ for carbon nuclei. The TOF counters measure the velocity $\beta=\mathrm{v} / \mathrm{c}$ and $|\mathrm{Z}|$ of cosmic rays. Two TOF planes are located above and two planes are located below the magnet. For $|\mathrm{Z}|=1$ particles, the average time resolution of each counter has been measured to be $160 \mathrm{ps}$ and the overall velocity resolution to be $\Delta \beta / \beta^{2}$ $=4 \%$. The time resolution improves for higher charges to reach $50 \mathrm{ps}$ for oxygen nuclei with the corresponding improvement in the velocity resolution of $\Delta \beta / \beta^{2}=$ $1 \%$. This discriminates between downward- and upwardgoing particles. The coincidence of signals from the four TOF planes provides a basis for charged particle trigger, whereas the coincidence of 3 out of the 4 TOF layers provides an unbiased trigger. The unbiased trigger, prescaled to $1 \%$, is used to measure the trigger efficiency from the data. To distinguish antiprotons and positrons from protons and electrons which are reconstructed in the tracker with wrong rigidity sign due to the finite tracker resolution or due to interactions with the detector materials, a charge confusion estimator, $\Lambda_{C C}$, is defined using the boosted decision tree technique. The estimator combines information from the tracker such as the track $\chi^{2} /$ d.o.f., rigidities reconstructed with different combination of tracker layers, the number of hits in the vicinity of the track, and the charge measurements in the TOF and the tracker. With this method, antiprotons/positrons have $\Lambda_{C C} \sim+1$ whereas charge confusion protons/electrons have $\Lambda_{C C} \sim-1$. This ensures efficient separation of the signal from the charge confusion events. The measured charge confusion probability is less than $1 \%$ up to $300 \mathrm{GV}$ and less than $10 \%$ up to TV. The TRD uses transition radiation to distinguish between $\bar{p}(\mathrm{p})$ and $\mathrm{e}^{-}\left(\mathrm{e}^{+}\right)$and $\mathrm{dE} / \mathrm{dx}$ to independently identify nuclei. It consists of 5248 proportional tubes of 6 $\mathrm{mm}$ diameter with a maximum length of $2 \mathrm{~m}$ assembled in 16-tube modules. The 328 modules are mounted in 20 layers. There are 12 layers of proportional tubes along the $\mathrm{y}$ axis located in the middle of the TRD and, along the $\mathrm{x}$ axis, four layers located on top and four on bottom. To differentiate between $\bar{p}(\mathrm{p})$ and $\mathrm{e}^{-}\left(\mathrm{e}^{+}\right)$in the TRD, signals from the 20 layers of proportional tubes are combined into a TRD estimator, $\Lambda_{T R D}$, formed from the ratio of the log-likelihood probability of the $\mathrm{e}^{ \pm}$hypothesis to that of the $\bar{p}(\mathrm{p})$ hypothesis in each layer. Antiprotons and protons have $\Lambda_{T R D} \sim 1$ whereas electrons and positrons have $\Lambda_{T R D} \sim 0.5$. This provides efficient separation of $\bar{p}(\mathrm{p})$ and $\mathrm{e}^{-}\left(\mathrm{e}^{+}\right)$. The TRD $\bar{p}(\mathrm{p})$ rejection at $90 \%$ selection efficiency for $\mathrm{e}^{-}\left(\mathrm{e}^{+}\right)$is larger than 700 up to $300 \mathrm{GV}$ and larger than
10 up to TV. The ring imaging Čerenkov detector measures velocity and $|\mathrm{Z}|$. It consists of two radiators, an expansion volume and a photodetection plane. The dielectric radiators induce the emission of a cone of Čerenkov photons when traversed by charged particles with a velocity greater than the velocity of light in the material. The central radiator is formed by 16 sodium fluoride, $\mathrm{NaF}$, tiles, each $85 \times 85 \times 5 \mathrm{~mm}^{3}$, with a refractive index $\mathrm{n}=1.33$. These are surrounded by 92 tiles, each $115 \times 115 \times 25 \mathrm{~mm}^{3}$, of silica aerogel with a refractive index $n=1.05$. This allows the detection of particles with velocities $\beta>0.75$ with the $\mathrm{NaF}$ radiator and $\beta>0.953$ with the aerogel radiator. The expansion volume has a distance along $\mathrm{z}$ of $470 \mathrm{~mm}$ and is surrounded by a high reflectivity mirror to increase detection efficiency. The photodetection plane is an array of 10880 photosensors with an effective spatial granularity of $8.5 \times 8.5 \mathrm{~mm}^{2}$. The sum of the signal amplitudes is proportional to $\mathrm{Z}^{2}$. The opening angle of the Cerenkov radiation cone is a measure of the velocity of the incoming charged particle. Typical velocity resolution is $\Delta \beta / \beta^{2}=0.1 \%$ for $|\mathrm{Z}|=1$. As an example, Fig. 2 shows clear separation of $|\mathrm{Z}|=1$ events measured by AMS in the $\left(\beta_{R I C H}-\operatorname{sign}(\mathrm{R}) \Lambda_{T R D}\right)$ plane for the absolute rigidity range 5.4-6.5 GV. The contributions of primary $\bar{p}, p, e^{+}, e^{-}$and secondary $\pi^{+}, \pi^{-}$from interactions within the detector, are clearly seen.

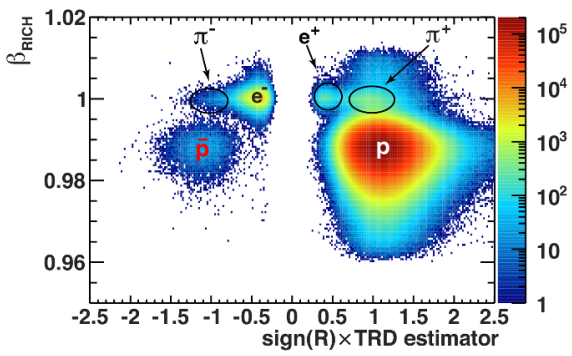

Figure 2. Example of negative rigidity and positive rigidity data samples in the $\left(\beta_{R I C H}-\operatorname{sign}(\mathrm{R}) \Lambda_{T R D}\right)$ plane for the absolute rigidity range $5.4-6.5 \mathrm{GV}$. The contributions of $\bar{p}, p, e^{+}, e^{-}, \pi^{+}, \pi^{-}$are clearly seen.

Finally, the three-dimensional imaging capability of the 17 radiation length ECAL allows for an accurate measurement of the $\mathrm{e}^{ \pm}$energy $\mathrm{E}$ and shower shape. It consists of a multilayer sandwich of 98 lead foils and $\sim 50000$ scintillating fibers with an active area of $648 \times 648 \mathrm{~mm}^{2}$ and a thickness of $166.5 \mathrm{~mm}$. The calorimeter is composed of 9 superlayers, with the fibers running in one direction only in each superlayer. The 3-D imaging capability of the detector is obtained by stacking alternate superlayers with fibers parallel to the $\mathrm{x}$ - and $\mathrm{y}$-axes ( 5 and 4 superlayers, respectively). The energy resolution has been measured to be $\sigma(E) / E=\sqrt{(0.104)^{2} / E+(0.014)^{2}}(\mathrm{E}$ in $\mathrm{GeV})$. The uncertainty of the absolute $\mathrm{e}^{ \pm}$energy scale has been verified to be $2 \%$ in the range $10-290 \mathrm{GeV}$. Below $10 \mathrm{GeV}$ it increases to $5 \%$ at $0.5 \mathrm{GeV}$ and above $290 \mathrm{GeV}$ to $4 \%$ at $700 \mathrm{GeV}$. To cleanly separate protons from electrons 
and positrons, an ECAL estimator, is constructed using the 3-D shower shape in the ECAL. The proton rejection power of the ECAL estimator when combined with the energy-momentum matching requirement $\mathrm{E} / \mathrm{p}>0.75$ reaches $\sim 10000$. The entire detector has been extensively calibrated in a test beam at CERN with $\mathrm{e}^{+}$and $\mathrm{e}^{-}$from 10 to $290 \mathrm{GeV} / \mathrm{c}$, with p at 180 and $400 \mathrm{GeV} / \mathrm{c}$, and with $\pi$ from 10 to $180 \mathrm{GeV} / \mathrm{c}$, which produce transition radiation equivalent to $\mathrm{p}$ up to $1.2 \mathrm{TeV} / \mathrm{c}$. Measurements with 18 different beam energies/particles scanning 2000 detector positions were performed. A Monte Carlo program based on the GEANT 4.10.1 package is used to simulate physics processes and signals in the detector.

\section{Elementary Particles in Cosmic Rays}

AMS has collected $1.07 \times 10^{11}$ cosmic rays in 6.5 years of operation. A total of 1.9 million positrons are identified in the energy range from $0.5 \mathrm{GeV}$ to $1 \mathrm{TeV}$ and this allows for a detailed study of the properties of the positron flux.



Figure 3. The fit of Eq.1 (green line) to the positron flux in the energy range $[1-1000] \mathrm{GeV}$ together with the $68 \% \mathrm{CL}$ interval (green band). The red dots represent the measured average positron flux values over the measurement period scaled by $E^{3}$. The source term contribution is represented by the magenta area, and the diffuse term contribution by the grey area

As seen in Fig. 3, the positron spectrum, scaled by $E^{3}$, exhibits a complex behavior. It decreases rapidly with energy below $\sim 7 \mathrm{GeV}$. In the energy range [7.10 - 27.25] $\mathrm{GeV}$ it is energy independent, with spectral index, $\gamma=-$ $2.986 \pm 0.005$, then it increases, with $\gamma=-2.716 \pm 0.037$, in the energy range [55.58 - 148.81] GeV. Above $148.81 \mathrm{GeV}$ the spectral index experiences significant decrease reaching $\gamma=-3.35 \pm 0.32$ in the highest energy range [290 $1000] \mathrm{GeV}$. This behavior is consistent with a new source of high energy positrons with a characteristic cutoff energy, whether of dark matter or astrophysical origin; it is not consistent with exclusive secondary production of positrons in collisions of cosmic rays.

Moreover it is possible to describe the positron spectrum with a minimal model. In this model the positron flux $\Phi_{e^{+}}$is parameterized as the sum of a diffuse term and a new source term:

$$
\Phi_{e^{+}}(E)=\frac{E^{2}}{\hat{E}^{2}}\left[C_{e^{+}}\left(\hat{E} / E_{1}\right)^{\gamma_{e^{+}}}+C_{s}\left(\hat{E} / E_{2}\right)^{\gamma_{s}} e^{\left(-\hat{E} / E_{s}\right)}\right]
$$

The diffuse term is a power law function, which is characterized by a normalization factor $\mathrm{C}_{e^{+}}$and a spectral index $\gamma_{e^{+}}$. The source term is a power law function with an exponential cutoff, which is characterized by a normalization factor $\mathrm{C}_{s}$, a spectral index $\gamma_{s}$ and a cutoff energy $\mathrm{E}_{s}$. In order to account for solar modulation effects the forcefield approximation is used, with the energy of particles in the interstellar space $\hat{E}=E+\psi_{e^{+}}$and the effective solar potential $\psi_{e^{+}}$accounting for the effects of the solar magnetic field. The constant $\mathrm{E}_{1}$ is chosen to be $7.0 \mathrm{GeV}$ to minimize the correlation between parameters $\mathrm{C}_{e^{+}}$and $\gamma_{e^{+}}$. The constant $\mathrm{E}_{2}$ is chosen to be $60.0 \mathrm{GeV}$ to minimize correlation between the parameters $\mathrm{C}_{s}$ and $\gamma_{s}$. A fit to the data in the energy range [1 - 1000] GeV accounts for a correlated systematic error due to the acceptance uncertainty. This correlated error is estimated to be $1 \%$ in the fit energy range, it affects only normalization parameters $\mathrm{C}_{e^{+}}$and $\mathrm{C}_{s}$ and is quoted as a systematic error on these two parameters. The fit yields $\chi^{2} /$ d.o.f. $=50 / 65$ and $\psi_{e^{+}}=1.10 \pm 0.04 \mathrm{GeV}$ for the solar modulation potential; $\mathrm{C}_{e^{+}}=(6.52 \pm 0.13 \pm$ $0.07($ syst $)) \times 10^{-2}\left[\mathrm{~m}^{2} \mathrm{sr} s \mathrm{GeV}\right]^{-1}, \gamma_{e^{+}}=-4.08 \pm 0.07$ for the diffuse term; and $\mathrm{C}_{s}=(6.80 \pm 0.14 \pm 0.07$ (syst) $) \times$ $10^{-5}\left[\mathrm{~m}^{2} \mathrm{sr} s \mathrm{GeV}\right]^{-1}, \gamma_{s}=-2.581 \pm 0.055$, and the inverse cutoff energy $1 / \mathrm{E}_{s}=1.22 \pm 0.35 \mathrm{TeV}^{-1}$ for the source term. The last parameter corresponds to $\mathrm{E}_{s}=820_{-180}^{+330} \mathrm{GeV}$. The result of the fit is superimposed in Fig. 3. As seen, the source term dominates the positron spectrum at energies above $20 \mathrm{GeV}$, the region where solar modulation effects are negligible. It is the contribution of the source term that leads to the observed increase of the positron spectrum in the energy range from 27.25 to $290 \mathrm{GeV}$. The decrease of the positron spectrum above $290 \mathrm{GeV}$ is very well described by the sharp exponential cutoff of the source term. Note that the choice of the $\mathrm{E}_{1}$ and $\mathrm{E}_{2}$ constants does not affect values and errors of the fit parameters $\psi_{e^{+}}, \gamma_{e^{+}}, \gamma_{s}$ and $1 / \mathrm{E}_{s}$, i.e. neither the shapes nor the magnitudes of the diffuse and the source terms shown in Fig. 3 depend on the choice of $E_{1}$ and $E_{2}$. The significance of the finite energy cutoff is established at a level of $3.5 \sigma$ from the errors on the $1 / \mathrm{E}_{s}$ fit parameter and this result is based on the positron data alone.

Another AMS measurement that is very interesting for dark matter indirect search is the antiproton ratio $\bar{p} / p$.

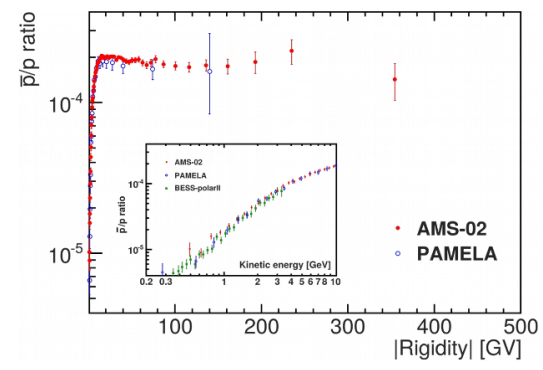

Figure 4. The $\bar{p} / p$ flux ratio as measured by AMS along with previous measurments. 
Overall, in the absolute rigidity range $1-450 \mathrm{GV}$ $3.49 \times 10^{5}$ antiproton events are selected. It is important to note that in the high rigidity range above $100 \mathrm{GV}$ AMS has selected $2200 \bar{p}$. This can be compared with $3 \bar{p}$ detected before AMS in the same rigidity range.

The $\bar{p} / p$ flux ratio as measured by AMS along with previous measurments is presented in Fig. 4. Above $\sim 60$ $\mathrm{GV}$ the ratio is found to be independent on rigidity. This observation caused a major revision of modeling cosmic ray antiprotons produced in ordinary collisions of protons with interstellar media (see e.g. [15-17]). The revised conservative models still predicts a $30-40 \%$ drop of the $\bar{p} / p$ ratio from the maximum, that is not supported by the AMS data, providing room for the presence of exotic/new antiproton source within the large existing theoretical uncertainties of secondary $\bar{p}$ production/propagation models.

It is interesting to compare behavior of the spectra of all elementary particles among themselves. Traditionally, electrons and protons are assumed to be primary cosmic rays, i.e. particles produced directly at sources of cosmic rays like exploding supernovae. On the contrary, positrons and antiprotons are assumed to be secondary cosmic rays, i.e. coming from the interaction of primary cosmic rays with the interstellar media. In addition, electrons and positrons have much smaller mass than protons and antiprotons so they lose much more energy in the galactic magnetic fields. This is illustrated in Fig. 5, where the behavior of the electron spectrum is compared with that of the proton, prositron and antiproton spectrum. As expected, in the rigidity region free of solar modulation effects, above $20 \mathrm{GeV}$, the proton spectrum is much harder that the electron spectrum. However, the behavior of antiprotons (assumed to be secondaries) and protons (assumed to be primaries) at high rigidities are very similar, while the behavior of electrons (i.e. primaries) and positrons (secondaries) are very different.

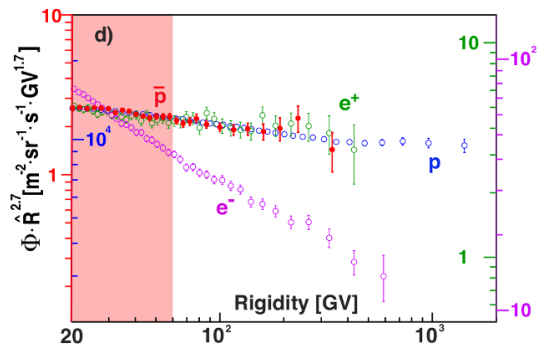

Figure 5. AMS measurements of the fluxes of elementary particles. The positron, antiproton and proton fluxes show the very same functional behavior in the absolute rigidity range 60-500 $\mathrm{GV}$, whereas electrons exhibit distinctly different dependence.

Most surprising is that above $60 \mathrm{GeV}$, positrons, protons and antiprotons display an identical energy dependence whereas electrons exhibit a totally different energy dependence. A compelling explanation for this AMS observation within traditional models of galactic cosmic rays is yet to be found.

\section{Conclusions}

In six years on the ISS, AMS has recorded more than 100 billion cosmic ray events. The latest AMS measurements of the positron spectrum and positron fraction, the antiproton/proton ratio, the behavior of the fluxes of electrons, positrons and protons provide precise and unexpected information on the production, acceleration and propagation of cosmic rays. The accuracy and characteristics of the data, simultaneously from many different types of cosmic rays require the development of a comprehensive model of cosmic rays. As a magnetic spectrometer studying cosmic rays, AMS is unique in its precision and energy reach. For the foreseeable future this is the only magnetic spectrometer in space to perform precision measurements and to explore the unknown with high expectations for exciting discoveries. Published data of AMS fluxes and flux ratios can be downloaded from the ASI/SSDC cosmic ray database [18].

\section{References}

[1] M. Aguilar et al. (AMS), Phys. Rev. Lett. 110, 141102 (2013)

[2] L. Accardo et al. (AMS), Phys. Rev. Lett. 113, 121101 (2014)

[3] M. Aguilar et al. (AMS), Phys. Rev. Lett. 113, 121102 (2014)

[4] M. Aguilar et al. (AMS), Phys. Rev. Lett. 113, 221102 (2014)

[5] M. Aguilar et al. (AMS), Phys. Rev. Lett. 114, 171103 (2015)

[6] M. Aguilar et al. (AMS), Phys. Rev. Lett. 115, 211101 (2015)

[7] M. Aguilar et al. (AMS), Phys. Rev. Lett. 117, 091103 (2016)

[8] M. Aguilar et al. (AMS), Phys. Rev. Lett. 117, 231102 (2016)

[9] M. Aguilar et al. (AMS), Phys. Rev. Lett. 119, 251101 (2017)

[10] M. Aguilar et al. (AMS), Phys. Rev. Lett. 120, 021101 (2018)

[11] M. Aguilar et al. (AMS), Phys. Rev. Lett. 121, 051103 (2018)

[12] M. Aguilar et al. (AMS), Phys. Rev. Lett. 121, 051101 (2018)

[13] M. Aguilar et al. (AMS), Phys. Rev. Lett. 121, 051102 (2018)

[14] M. Aguilar et al. (AMS), Phys. Rev. Lett. submitted (2018)

[15] N. Tomassetti, Phys. Rev. D 92, 081301 (2015)

[16] J. Feng, N. Tomassetti, A. Oliva, Phys. Rev. D 94, 123007 (2016)

[17] N. Tomassetti, J. Feng, A. Oliva, Research Notes of the AAS 1, 35 (2017)

[18] (ASI/SSDC cosmic ray database https://tools.ssdc.asi.it/CosmicRays/) 